\title{
Comparison between developments of emergency medicine services in Saudi Arabia, compare to USA and Canada
}

\author{
Zohair Al Aseri* \\ Departments of Emergency Medicine and Critical Care, King Saud University Medical City, Riyadh, KSA
}

\begin{abstract}
Objective: The aim of this research is to compare the development of emergency medicine in USA, Canada and Saudi Arabia. It is further aimed to identify the sort of measurements and procedures those are followed in Saudi Arabia, USA and Canada.

Method: The specific peer reviewed studies have been selected in this research by the help of different electronic databases including Science Direct, MEDLINE and CINAHL. The articles are included and excluded by the examination of the research quality, its significance with the research question and the publication year.

Result: There are five themes that have been extracted from the research articles including the development of emergency medicine in Saudi Arabia and United States, development of emergency medicine in Saudi Arabia and Canada, healthcare procedure and measurements in USA, healthcare procedure and measurements in Canada and the healthcare procedure and measurements in Saudi Arabia.
\end{abstract}

Conclusion: It can be concluded that there are several differences in the emergency medicine procedures of the three countries and they use different methods, which ensure in providing the quality care to the patients.

\section{Abbreviations}

EMS: Emergency Medical Services; ED: Emergency Department; MVC: Motor Vehicle Collision; CDC: Centre for Disease Control and Prevention; ICD: International Classification of Diseases; CIHI: Canadian Institute for Health Information; CED-DxS: Canadian Emergency Department Diagnosis Shortlist.

\section{Introduction}

\section{Background of the study}

The emergency medical services are known as EMS which is a significant public service. The emergency medical services can be recognized easily when the medical helicopters and the ambulances are responding to different incidents in the area. However, the emergency medical service is a vast field apart from the transport and medical response. It is mainly a part of the fundamental system of different agencies and organizations along with the transportation and communication network. This system further includes the specialty care centers, trauma centers, rehabilitation facilities, trained professionals, hospitals, nurses, therapists, government officials, and the administrators. This system contains the informed public, nurses and the pre-hospital personnel who know about the situation of medical emergency. All the people involved in the emergency medical services have a significant job to become the part of a coordinated care system. There is a complicated interaction of the fundamental components of the EMS system in the management of a critical and single incident. The structure of emergency medical services is different in various regions and communities. The services of pre-hospital can be hospital based, fire based or the public safety companies. However, the components of emergency medical services remain same [1]. According to Heyworth (2011), emergency department (ED) is thought to be in a major dysfunction due to inadequate ED capacity, outdated design of ED, junior doctor-based workforce as well as traditional ED processes [2].

The emergency medical services provide the acute medical care to different types of serious emergencies like the allergic reactions, poisoning due to chemicals and drugs, accidents including fractures, respiratory or cardiac failure, child abuse and other conditions [3]. The team of emergency medical services contains those people who are expert in the providing the emergency medical services, ambulance employees, fire fighters, and the paramedics. The level of services consists of three different categories including the basic life support, care by healthcare professionals and the advanced life support. The nurses and physicians are rarely available to provide the pre hospital emergency care. Many of the emergencies are managed by the paramedics and other people including the technician and the driver [4].

The paramedics are the trained healthcare professionals who respond to the patients in the medical emergency. They provide the assessment apart from the inpatient care along with the care and treatment. There are different levels of the paramedic practice and it is determined by the employing authority to determine the allotment up to the specified care level. The paramedics get appropriate training related to the tasks which they need to carry out. Nevertheless, they can face different obstacles and barriers in the discharge of their duties [4].

Correspondence to: Zohair Al Aseri MD, FRCPC (EM \& CCM), Department of Emergency Medicine and Critical Care, King Saud University Medical City, Riyadh, KSA, Tel: +96614671955; E-mail: zalaseri@ksu.edu.sa

Key words: emergency medicine, Saudi Arabia, healthcare, therapists, toxicology, ambulance

Received: March 03, 2017; Accepted: April 03, 2017; Published: April 06, 2017 


\section{Importance of the study}

The government of Saudi Arabia aims to improve the health care system of Saudi Arabia and want to provide the citizens with the possible care. The emergency medicine in Saudi Arabia are improving in a remarkable manner [5]. In the year 2004, the number of Saudi qualified physicians was four [6]. In the year 2013, the number of Saudi qualified emergency medicine is about 70 physicians who have acquired the training in Europe, North America, Australia and in Saudi Arabia [6]. There is a residency training program of emergency medicine in Saudi Arabia which provides training to about 50 people. In addition, there are various national and international fellowships which are related to the emergency medicine including critical medical care, international emergency medicines, disaster medicine, and toxicology [6].

The emergency medicine of United States and Canada are well developed. In the year 2010, the Royal College of surgeons and physicians of Canada obtained a new set of training for the emergency medicine. These guidelines have resulted in several changes to the residency training including the clinical time that has been spent in the emergency department and an increased structured approach to the post graduate students. It has been reported that the emergency medicine experts in Canada should obtain the expertise for about six months before providing the services of emergency medicine. The emergency medicine activities must also be structured, and identifiable with the measureable goals and objectives [7].

\section{Problem statement}

Saudi Arabia is an exclusive country which provides services of healthcare and it faces a biggest challenge in terms of healthcare. The challenge is that the Saudi Arabia experiences a huge gathering in the season of Hajj. It requires the healthcare provider to be competent enough in the disaster and emergency medicine [8]. The Ministry of Health must identify that the development of the possible healthcare services needs to provide the good strategy, vision, and the applicable plan. The government of Saudi Arabia has ordered to create a policy and procedure for the development of emergency medicine department. An important challenge that is faced by the health care system of Saudi Arabia is the motor vehicle collision MVC. The most common causes of hospital admission following injury were MVC and pedestrian injuries [9].

\section{Goals}

The main aim of this review is:

1. To compare the development of emergency medicine in Saudi Arabia, USA and Canada.

2. To identify different measurements and procedures being followed in Saudi Arabia, USA and Canada.

\section{Research question}

1. How Emergency Medicine is developing in USA, Canada and Saudi Arabia?

2. What are the different procedures and measurements followed in USA, Canada and Saudi Arabia in emergency medicine?

\section{Methods}

\section{Search strategy}

In this review article, the suitable peer reviewed studies are selected by the help of various electronic databases including MEDLINE, CINAHL and Science Direct. The overall search for this review included various keywords like "emergency medicine", "emergency medicine procedures", "emergency medicine measurements", "emergency medicine development", "emergency medicine in Saudi Arabia", "emergency medicine in USA" and "emergency medicine in Canada".

The inclusion and exclusion of the selected research articles is sometimes examined by the assessment of the research quality, its significance with the research question and the year of publication. The research articles consisted of different peer reviewed journals which were published in English and the different studies in relevance with the research questions. The studies of more than ten years old were not included. In addition, the studies which were selected had the quantitative and qualitative research design. The unpublished data and the incomplete manuscript were not included in the review. In addition, the articles which were not according to research aims were not included and the studies other than the English language are also excluded.

\section{Results}

\section{Development of emergency medicine in Saudi Arabia and United States}

The emergency medicine characterizes a new specialty and the United States is included among the countries to develop and identify emergency medicine training programs [10]. In Saudi Arabia the training of emergency medicine was developed in the year 2000 by the newly graduated physicians who finished their training in North America [11]. About $40 \%$ of the residents of Saudi Arabia have identified that their training program is not properly providing the preparation for the written and oral board exam. The lack of training can be due to the easy accessibility of the multiple educational resources. It is also due to the point of views of the Saudi residents that the faculty is not interested in the education of residents [12]. The deficiency of training can be affected by the lack of easy access to the different educational resources [11].

Another research finding is there was an increase in the number of patients seen per hour by the Saudi residents as compared to the US residents. The restrictions of duty hours and the guidelines of supervision in United States can limit the practice of the junior residents in the United States. It results in decreasing the ratio of average patients each hour. The importance of total number of the supervised patients in the success of a resident is not clear in obtaining the competency. It is further suggested that many patients experience the use of deliberate practice in providing care. It further provides the education for the development of competency and expertise. In the consideration of this problem, the Saudi training program facilitates in providing the patient care experience to the trainees [12].

It is felt by the Saudi trainees that their training program do not offer the appropriate education opportunities in order to become competent by performing the procedures which are not common. These procedures include vaginal deliveries, cricothyrotomy, cardiac pacing, and peri-cardiocentesis. This condition can be explained by restricting the ability of the simulation labs. There are about $63.3 \%$ of the Saudi residents who cannot access the simulation labs. It is further reported that there is some neutral response when the Saudi residents were asked about the management of trauma patients and the relevance of weekly academic activity to the daily clinical practice. There are two studies of the weekly academic activities which included the 
emergency medicine. It was reported in the research that involvement in the simulation produces a greater effect on the behavior, skills, and knowledge outcomes of the different participating residents [13].

The assessment of the residents and the feedback of the faculty are considered to be an important element in the improvement of the quality of medical training in United States. These successful programs are used as a tool in order to understand and listen to the perspective of trainees. As compared to the group in United States, there are a few residents in Saudi Arabia who have the ability to evaluate the faculty at least once in a year. The evaluation culture and the feedback can be different for the residents of Saudi Arabia. It cannot be considered as the major element in the residency training system [12]. However, the initial data can facilitate in providing some discussion areas and comparison for the future studies.

\section{Development of emergency medicine in Saudi Arabia and Canada}

In Saudi Arabia, an increase in the utilization of the emergency departments results in overcrowding. The citizens of Saudi Arabia have free medical care with a network of primary center of healthcare with the developing emergency care service [16]. Similarly, a research of Canada was carried out to identify the current condition of the emergency medical services along with the focus on the barriers. The research identified that there is a need of additional research education in the emergency medicine services [15]. Similarly, the Saudi study identified that the emergency departments are non urgent which can result in increasing the waiting times and delaying the interventions for the ill patients [16]. Conversely, there is a need to create an infrastructure for the advancement of the Canadian emergency medical services. In addition, the complications of the involvement of the emergency medical services must be addressed [15]. The research in Saudi Arabia explored the trends of using the emergency department over a period of three years. It was identified that the number of visits is increasing by $30 \%$ and $60 \%$ of the patients are sent to the hospital with the non urgent conditions. These further results in increasing the length of stay and many patients experience to visit the emergency department [17]. The Canadian research has been designed as the initiating point for the increased consensus driven methodology and gives an important direction for the round table discussions and survey. The research of Dainty et al.[15] had the strength of initiating a wide initiative including the national research agenda with the research methodology of qualitative research. This research has resulted in providing the various perspectives.

There is an increase in the evidence of development of emergency medicine in Saudi Arabia. It is necessary to examine the factors which affect the preferences of the patients for going to the emergency department with the non urgent problems [18]. Dainty et al.[15] has conducted the research to identify the Canadian experiences and perceptions which serve as an important stage in the building of research agenda for the emergency medical services. Similarly, different middle east studies identified that the emergency department care can be affected by younger age, unavailability of the physician or the healthcare source, and the negative point of views about the care providers $[19,20]$.

However, all of these factors cannot be generalized according to the unique types of the Middle East healthcare system. It has been suggested that the emergency medication system can be advanced by increasing the trust of patients in the different primary care services, providing the 24 hours quality care to the patients and advancing the system of laboratory investigations for the patients [21]. The study of Dainty et al.[15] contained a rigorous approach to conduct the qualitative data collection by including the analysis and interviews without any affiliations and ties to the emergency medicine clinical research community. It has further focused on analyzing the emerging themes in comparison with the predefined framework. The sample of this research was selected from a large group of individuals which have been involved actively in the research.

\section{Limitations}

The most important and significant research constraint in conducting the study is time. Another major constraint in the review includes the financial resources.

\section{Discussion}

\section{Healthcare procedure and measurements in USA}

The centre for disease control and prevention (CDC) has indicated that there is an increase in the demand of emergency services in the United States. The data of year 2009 has identified that there have been 124 million visits of emergency department by the patients which is higher as compared to year 1996 in which the number of visited people was found to be 90.5 million. There was an average of 42 visits in the 100 people as compared to the 35 visits per 100 people in the year 1996. There is greater increase in the emergency services of the elderly people because their number of visits per 100 people is 60 [21]. This condition is not due of the lack of primary care because majority of the people are required to be consulted in the emergency department. Many of the procedures and systems are being used for providing the emergency healthcare in the United States.

The root cause analysis is a technique which is used in engineering and it is similar to the technique of critical incident [22,23]. It is an investigation and problem solving approach which is mainly focused on the identification and understanding of the underlying causes of an event and the potential events that can be intercepted. The root cause analysis is performed in all the sentinel events in the United States, on the basis of the results of analysis, the organization is required to develop and carry out an implementation of the action plan in order to decrease the future risks and events [24].

The use of a qualitative process indicates that the main of root cause analysis in to identify an underlying cause of a healthcare condition by focusing on the enabling factors, latent conditions and the situational factors. Those people who are involved in a process of investigation ask different questions to the patients or the family members. The answers related to all of these questions facilitate in the identification of the causes of problems and the different safety barriers so that the similar problems can be easily prevented in the future. It is also important to consider that the events are immediately prior to the factors which result in causing an incidence [25]. The last step of the traditional root causes analysis in the development of recommendations for the process improvement on the basis of the findings of investigations [25].

\section{Healthcare procedure and measurements in Canada}

The combination of increased numbers of the admitted patients and an increase in the patient volume in the emergency departments results in increasing the time of stay in the department. An appropriate explanation of the patients who are looking for the emergency department medical care can result in understanding the function and use of emergency procedures and medicine [26,27]. Many of the 
authorities have been developed in Canada to develop the policies of improvement of healthcare in the emergency department. The collection of population based data has proven to be helpful in providing the quality health care because it facilitates in the identification of the prevailing trends of various health conditions in the population [28].

The triage acuity diagnosis, score, and the chief complaint of the patient are the important elements which facilitate in characterizing the illness burden in the emergency department of Canada [29]. The Canadian Emergency Department Information System and the Canadian Emergency Department Triage and Acuity Scale are included in the national standards for the data elements. Various diagnostic lists have been developed in the other countries and they facilitate in providing the quality health care [30]. In addition, there are no diagnostic lists present in Canada which can be used easily in the real time [31].

The $10^{\text {th }}$ revision of the International Classification of Diseases (ICD) with enhancement of Canada consists of 14203 diagnoses and it is known as the gold standard for the diagnostic coding. The use of complete ICD list in the real time emergency departments in not practical or cost effective. In Alberta and Ontario, the Canadian Institute for Health Information (CIHI) needs professional coding of the visits by using the complete ICD-10-CA. However, there are a few health information managers in Canada which provide an important barrier for the implementation of the health policy across the country [32]. The coding of emergency visits by using the retrospective charts must be reviewed and it facilitates in providing efficient healthcare to the patients. Gorelick [31] identified that many of the diagnoses are obtained from the administrative and the clinical sources which fall under the diagnostic group.

A diagnostic short list has been developed with about 837 items and it is the subset of complete ICD-10-CA. These lists have been developed by the expert consultation of the emergency medicine physicians across the Canada. In addition, the healthcare professionals have been working with the representatives of ICD and CIHI in order to ensure the widespread adoption. There was no standardized Canadian list previously [31]. Many other countries including France and Australia have developed their own specific lists of emergency departments [32].

These lists significantly overlap with each other and they are also different in a substantial manner by rendering the unique style to their needs and national settings. Many of the countries are also developing the emergency department specific shortlists of diagnosis which are according to the needs of participants and the population. The Canadian Emergency Department Diagnosis Shortlist (CED$\mathrm{DxS}$ ) is the initial emergency department diagnosis list which has been specifically developed for the Canadian emergency departments [32].

\section{Healthcare procedure and measurements in Saudi Arabia}

The emergency medical services in Saudi Arabia are being provided effectively by paramedics. The primary task of various paramedics is to cover the distance between the emergent conditions and patients, no matter how complicated the track shall be. It is thought to be a hard expedition which causes numerous difficulties. The answer to the traffic congestion question which causes numerous hurdles for paramedics is thought to be right which indicates to the fact that the congestion of traffic often interfere with the paramedic efficiency. In various countries, few reports indicate that there are street violence, insurgencies, riots and strikes which tend to block the roads and interfere with the paramedics working. Conversely, the congestion of traffic is overwhelmed in Saudi Arabia with heavy traffic and numerous different accidents such as VIP movements, traffic regulations brunt, signals, road repairs, and accident, police vigilance points to examine relevant papers, that tend to make the movements extremely hard and often interfere with the emergency medicine personnel performance comprising of paramedics and also endanger the patient's life [4].

The road accident sites are usually overcrowded with victim's relatives, friends, bystanders, and onlookers. Such kind of congregation is a huge cause for delay in the paramedic working. This response indicates that the queries related positively indicating the fact that smooth duty discharge by various paramedics is hard ${ }^{3}$. Moreover, it often causes risk and suffocation to the victim' life because of hypercapnia and asphyxia-related hypoxia. On the query of the association between the health professional's efficiency, paramedics' failures and administrations of EMS, the responded gave a positive response. However, in this subject the health care providers are considered to possess sound knowledge, but, the EMS is a matter of experience and training in both the advance and basic procedures of life support in which the providers are not competent enough [3].

\section{Conclusion}

The government of Saudi Arabia has an aim to improve the healthcare system of Saudi Arabia and they want to provide the people with improved care. The services in Saudi Arabia are improving these days in a remarkable manner. There is a need of improvement of emergency condition in Saudi Arabia as compared to the United States and Canada. The root cause analysis is an important technique which is being used in the United States. Canada has developed its own diagnostic list which serves to be an instrument for the development of diagnosis of disease.

\section{Recommendations}

When the training of emergency medicine is initiated in Saudi Arabia, there are certain areas which need improvement in the development of the emergency medicine. It includes the execution of the equipped simulation laboratories in the emergency medicine residency program with an opportunity for the trainees to carry out the procedures that are not common and conduct the resuscitation in the management of difficult cases. Moreover, it is appeared that the accessibility of the residents to various ebooks, journals and media must be increased. It is further believed that the faculty development must be increased to enhance the interest of faculty, faculty must be educated regarding the bedside teaching and the feedback process must be increased.

Grant: No financial support to be acknowledged.

Conflicts of interest: The author declares no conflicts of interest.

Ethics approval: Not applicable.

Author contribution: The author conceived the study, designed the review, and obtained research approval, drafted the manuscript, and contributed substantially to its revision.

\section{References}

1. EMS (2007) Emergency Medical Service, 24/ 7 Care Everywhere, National Highway Traffic Safety Administration.

2. Heyworth J (2011) Emergency medicine-quality indicators: the United Kingdom perspective. Acad Emerg Med 18: 1239-1241. [Crossref]

3. Bigham BL, Buick JE, Brooks SC, Morrison M, Shojania KG, et al. (2011) Patient 
safety in emergency medical services: a systematic review of the literature. Prehospital Emergency Care 16: 20-35.

4. Alanazi AF (2012) Emergency medical services in Saudi Arabia: A study on the significance of paramedics and their experiences on barriers as inhibitors of their efficiency. Int J Appl Basic Med Res 2: 34-37.

5. Almalki M, Fitzgerald G, Clark M (2011) Health care system in Saudi Arabia: an overview. East. Mediterr. Health J 17(10): 784-793.

6. MH (2013) Guidelines for Emergency Department, Ministry of Health, Kingdom of Saudi Arabia.

7. Sagarin MJ, Barton ED, Chng YM, Walls RM, National Emergency Airway Registry (NEAR) Investigators (2005) Airway management by US and Canadian emergency medicine residents: A multicenter analysis of more than 6,000 endotracheal intubation attempts. Ann Emer Med 46: 328-336.

8. Memish ZA, McNabb SJN, Mahoney F, Alrabiah F, Marano N, et al. (2009) Establishment of public health security in Saudi Arabia for the 2009 Hajj in response to pandemic influenza AH1N1. The Lancet 374: 1786-1791.

9. Al-Habib A, A-shail A, Alaqeel A, Zamakhshary M, Al-bedah K, et al. (2013) Causes and patterns of adult traumatic head injuries in Saudi Arabia: implications for injury prevention. Ann Saudi Med 33: 351-355.

10. Alagappan K, Holliman CJ (2005) History of the development of international emergency medicine. Emerg Med Clin North Am 23: 1-10. [Crossref]

11. Qureshi N (2009) International Perspective from Saudi Arabia on "Procedural Skills Training During Emergency Medicine Residency: Are We Teaching the Right Things?" West J Emerg Med 10: 157. [Crossref]

12. Alghamdi K, Alburaih A, Wagner MJ (2014) A Comparison between Emergency Medicine Residency Training Programs in the United States and Saudi Arabia from the Residents' Perception. Emerg Med Int: 362624. [Crossref]

13. McGaghie WC, Issenberg SB, Cohen MER, Barsuk JH, Wayne DB (2011) Does simulation-based medical education with deliberate practice yield better results than traditional clinical education? A meta-analytic comparative review of the evidence. Acad Med 86: 706-711. [Crossref]

14. Sun BC, Hsia RY, Weiss RE, Zingmond D, Liang LJ, et al. (2013) Effect of emergency department crowding on outcomes of admitted patients. Annals of Emergency Medicine 61: 605-611.

15. Dainty KN, Jensen JL, Bigham BL, Blanchard IE, Brown LH, et al. (2013) Developing a Canadian emergency medical services research agenda: a baseline study of stakeholder opinions. CJEM 15: 83-89. [Crossref]

16. Alyasin A, Douglas C (2014) Reasons for non-urgent presentations to the emergency department in Saudi Arabia. Int Emer Nur 22: 220-225.

17. Rehmani R, Norain A (2007) Trends in emergency department utilization in a hospital in the Eastern region of Saudi Arabia. Saudi Med Jour 28: 236-240.
18. Pines JM, Hilton JA, Weber EJ, Alkemade AJ, Al Shabanah H, et al. (2011) International perspectives on emergency department crowding. Academic Emergency Medicine 18: 1358-1370.

19. Carret MLV, Fassa ACG, Domingues MR (2009) Inappropriate use of emergency services: a systematic review of prevalence and associated factors. Cadernos Saude Publica 25: 7-28. [Crossref]

20. Jerius M, El-Salim A, Tarawneh MD, Habashneh M (2010) Inappropriate utilization of emergency medical services at Prince Ali Military Hospital. Jour Royal Med Serv 17: $32-35$.

21. Cai R, Crane E, Poneleit K, Paulozzi L (2010) Emergency department visits involving nonmedical use of selected prescription drugs in the United States, 2004-2008. Journal of Pain and Palliative Care Pharmacotherapy 24: 293-297.

22. Schluter J, Seaton P, Chaboyer W (2008) Critical incident technique: a user's guide for nurse researchers. Jour Adv Nurs 61: 107-114.

23. Morrow D, North R, Wickens CD (2005) Reducing and mitigating human error in medicine. Reviews of Human Factors and Ergonomics 1: 254-296.

24. Mills PD, Neily J, Luan D, Stalhandske E, Weeks W (2005) Using aggregate root cause analysis to reduce falls and related injuries. Joint Commission Journal on Quality and Patient Safety 31: 21-31.

25. Rothschild JM, Landrigan CP, Cronin JW, Kaushal R, Lockley SW, et al. (2005) The Critical Care Safety Study: The incidence and nature of adverse events and serious medical errors in intensive care. Criti Care Medi 33: 1694-1700.

26. Haggerty J, Burge F, Lévesque JF, Gass D, Pineault R, et al. (2007) Operational definitions of attributes of primary health care: consensus among Canadian experts. Annals of Family Medicine 5(4), 336-344.

27. Ayatollahi H, Bath PA, Goodacre S (2009) Accessibility versus confidentiality of information in the emergency department. Emergency Medicine Journal 26: 857-860.

28. Grafstein E, Bullard MJ, Warren D, Unger B (2008) Revision of the Canadian Emergency Department Information System (CEDIS) Presenting Complaint List version 1.1. CJEM 10: 151-173.

29. May LS, Griffin BA, Bauers NM, Jain A, Mitchum M, et al. (2010) Emergency department chief complaint and diagnosis data to detect influenza-like illness with an electronic medical record. West J Emerg Med 11: 1-9. [Crossref]

30. Heeks R (2006) Health information systems: Failure, success and improvisation. Int $J$ Med Inform 75: 125-137.

31. Gorelick MH, Knight S, Alessandrini EA, Stanley RM, Chamberlain JM, et al. (2007) Lack of agreement in pediatric emergency department discharge diagnoses from clinical and administrative data sources. Acad Emerg Med 14: 646-652.

32. Unger B, Afilalo M, Boivin JF, Bullard M, Grafsteiin E, et al. (2010) Development of the Canadian emergency department diagnosis shortlist. CJEM 12: 311-319.

Copyright: $\odot 2017 \mathrm{Al}$ Aseri Z. This is an open-access article distributed under the terms of the Creative Commons Attribution License, which permits unrestricted use, distribution, and reproduction in any medium, provided the original author and source are credited. 